PROCEEDINGS OF THE

AMERICAN MATHEMATICAL SOCIETY

Volume 137, Number 2, February 2009, Pages 751-759

S 0002-9939(08)09584-1

Article electronically published on August 28, 2008

\title{
FUNCTION SPACES OF CW HOMOTOPY TYPE ARE HILBERT MANIFOLDS
}

\author{
JAKA SMREKAR AND ATSUSHI YAMASHITA
}

(Communicated by Alexander N. Dranishnikov)

\begin{abstract}
Let $X$ be a countable CW complex and $Y$ an ANR (for metric spaces) and let $Y^{X}$ denote the space of continuous maps from $X$ to $Y$ with the compact-open topology. We show that, under mild restrictions, the following are equivalent: (1) $Y^{X}$ is an $\ell^{2}$-manifold, (2) $Y^{X}$ is an ANR, (3) $Y^{X}$ has the homotopy type of a $\mathrm{CW}$ complex. We also give a few interesting examples and applications.
\end{abstract}

\section{INTRODUCTION}

Sakai 14, paragraph following 'MAIN ThEOREM'] showed that if $X$ is a nondiscrete compactum and $Y$ is a separable completely metrizable ANR with no isolated points, then the space $Y^{X}$ of continuous maps $X \rightarrow Y$ with the compact open topology is an $\ell^{2}$-manifold.

There are obstructions to generalizing that to noncompact domain spaces. An evident one is that if $Y^{X}$ is an $\ell^{2}$-manifold, it has to be an ANR. This in turn implies that $Y^{X}$ has the homotopy type of a CW complex; see Milnor [12, Theorem 2]. In general, these implications are not equivalences. As an example of the latter, the cone of any metric non-ANR compactum is contractible but is not an ANR.

When $X$ is not a compactum, $Y^{X}$ can easily lack the 'nice' local properties of ANRs. For example, taking $Y$ to be the unit circle $S^{1}$, and $X$ the countable planar CW complex $\bigcup_{n \in \mathbb{Z}}\left\{(x, y) \in \mathbb{R}^{2} ;(x-2 n)^{2}+y^{2}=1\right\}$, we note that $Y^{X}$ is not locally path connected. In particular, it is not an ANR and in fact does not have the homotopy type of a CW complex, although it is a metrizable topological group.

If $X$ is a countable CW complex and $Y$ is an ANR, then it turns out that $Y^{X}$ is an ANR if and only if it has the homotopy type of a CW complex. Assuming Sakai's conditions on $X$ (discrete) and $Y$ (separable and complete with no isolated points), the two are equivalent to $Y^{X}$ being an $\ell^{2}$-manifold.

Received by the editors February 1, 2008.

2000 Mathematics Subject Classification. Primary 54C35; Secondary 55M15, 57N20.

The first author was supported in part by the ARRS research project No. J1-6128-0101-04.

The second author was supported by Research Fellowships of the Japan Society for the Promotion of Science for Young Scientists.

(C)2008 American Mathematical Society Reverts to public domain 28 years from publication 


\section{Statements of the MAin Results}

Recall that a metrizable space $Z$ is an absolute neighborhood retract (ANR) for metric spaces, which hereafter we refer to simply as an ANR, if for every closed embedding $Z \hookrightarrow W$, where $W$ is metric, the image of $Z$ is a retract of a neighborhood in $W$.

Also, we say that $Z$ has the homotopy type of $W$ if $Z$ and $W$ are homotopy equivalent, that is, if there exist continuous functions $f: Z \rightarrow W$ and $g: W \rightarrow Z$ such that the composites $g \circ f$ and $f \circ g$ are homotopic to their respective identities.

Finally, $Z$ is semilocally contractible if each point has a neighborhood that is contractible within $Z$.

Theorem 1.1. Let $X$ be a countable $C W$ complex and let $Y$ be an $A N R$. The following are equivalent.

(i) The space $Y^{X}$ is an $A N R$.

(ii) The space $Y^{X}$ has the homotopy type of a $C W$ complex.

(iii) The space $Y^{X}$ is semilocally contractible.

The first author has investigated conditions under which $Y^{X}$ has CW homotopy type; see [15], 16], and [17]. Here we can say that there exist nontrivial (families of) examples of infinite complexes $X$ and interesting ANR spaces $Y$ for which the function space $Y^{X}$ has $\mathrm{CW}$ homotopy type (and is hence an ANR). This makes the above theorem nonfrivolous.

On the other hand, there seems to be no hope in trying to completely characterize the question of CW homotopy type of the function space $Y^{X}$ in terms of properties of $X$ and $Y$, as some special cases reduce to very difficult open problems in topology; the interested reader is referred to [17].

In his paper [21], the second author considers spaces of functions defined on a noncompact space equipped with the uniform topology. There he gives a condition under which such a function space is a topological manifold locally modeled on a (nonseparable) Hilbert space. In the present paper we prove a result in this direction for the compact-open topology. Namely, we show that if $Y$ is a separable complete metric space, except for trivial cases, the equivalent conditions of Theorem 1.1 are equivalent to that $Y^{X}$ is such a manifold, where the model is the separable Hilbert space.

Theorem 1.2. Let $X$ be a countable $C W$ complex and let $Y$ be a separable completely metrizable space. Assume that $\operatorname{dim} X \geq 1$ and that $Y$ has no isolated points. Then $Y^{X}$ is an $\ell^{2}$-manifold if and only if one (and hence all) of the conditions (i)-(iii) in Theorem [1.1] hold.

In general, for a metrizable space $E$, by an $E$-manifold we mean a metrizable space $M$ such that each point of $M$ has an open neighborhood homeomorphic to some open set in $E$. If we let $E=\ell^{2}$, we obtain the notion of an $\ell^{2}$-manifold, where $\ell^{2}$ is the separable real Hilbert space of square summable sequences.

Remark 1.3. Theorem 1.2 does not generalize to the nonseparable range space $Y$. Indeed, there exists a nonseparable AR $Y$ such that $Y^{X}$ is not homogeneous for any compact metric space $X$. Fix an uncountable set $\Lambda$ and let $\ell^{1}(\Lambda)$ be the Banach space of functions $x: \Lambda \rightarrow \mathbb{R}$ such that $\sum_{\lambda \in \Lambda}|x(\lambda)|<\infty$. Define $e_{\lambda} \in \ell^{1}(\Lambda)$ by $e_{\lambda}(\mu)=0$ whenever $\mu \neq \lambda$ and $e_{\lambda}(\lambda)=1$. Define $Y$ as the union $Y=\bigcup_{\lambda \in \Lambda}\left[0, e_{\lambda}\right]$ of intervals, regarded as a topological subspace of $\ell^{1}(\Lambda)$. Let $X$ be a compact 
metric space and for $y \in Y$, denote by $c_{y}$ the constant map from $X$ to $y \in Y$. Then $c_{e_{\lambda}}$ has a separable neighborhood $\left(0, e_{\lambda}\right]^{X}$ in $Y^{X}$ for each $\lambda$ (see Engelking [5, Theorem 3.4.16]), but every neighborhood of $c_{0}$ has an uncountable discrete closed subset (consisting of constant maps). This implies that $Y^{X}$ is not homogeneous.

For function spaces $Y^{X}$ satisfying the hypotheses of Theorem 1.2, the $\ell^{2}$-manifold structure of $Y^{X}$ imposes a certain rigidity on its topology; namely:

Corollary 1.4. Let $X$ and $X^{\prime}$ be homotopy equivalent countable $C W$ complexes, each of dimension at least 1 , and let $Y$ and $Y^{\prime}$ be homotopy equivalent separable completely metrizable ANRs without isolated points. Assume that $Y^{X}$ has $C W$ homotopy type. Then $Y^{X}$ and $Y^{\prime X^{\prime}}$ are homeomorphic.

We discuss only the case where the domain complex $X$ is countable. To see why this is justified, note first that if $Y^{X}$ is an ANR, then it must be metrizable. The next lemma shows that if $Y^{X}$ is metrizable, then essentially $X$ must be countable:

Lemma 1.5. Assume that $X$ is a $C W$ complex and that $Y$ is a space having a nontrivial path component. If $Y^{X}$ is metrizable, then $X$ is countable.

\section{Proof of Theorem 1.1 and Lemma 1.5}

For spaces $X, Y$ and subsets $A \subset X, B \subset Y$, let

$$
(Y, B)^{(X, A)}=\left\{f \in Y^{X} ; f(A) \subset B\right\} .
$$

Then the subsets of the form $(Y, V)^{(X, K)}$, where $K$ is compact and $V$ is open, constitute a subbasis for the compact-open topology of $Y^{X}$.

The main ingredient of the proof of Theorem 1.1 is the following characterization of ANR spaces conjectured by R. Geoghegan and proved by R. Cauty:

Theorem 2.1 (Cauty 2], 'Théorème'). A metrizable space $Z$ is an ANR if and only if every open set of $Z$ has the homotopy type of a $C W$ complex.

An open covering $\mathcal{U}$ of $Z$ is called numerable if a locally finite partition of unity is subordinate to $\mathcal{U}$. Recall that every open covering of a paracompact space is numerable.

Proof of Theorem 1.1. The implication (i) $\Longrightarrow$ (ii) is contained in Theorem 2.1. while (ii) $\Longleftrightarrow$ (iii) follows from [16, Theorem 2.2.1(iv)].

To prove (ii) $\Longrightarrow$ (i), assume that $Y^{X}$ has CW homotopy type. By Theorem 2.1, it suffices to show that every open set of $Y^{X}$ has the homotopy type of a CW complex. Let $\mathcal{B}$ be the collection of open sets of $Y^{X}$ of the form $\bigcap_{i=1}^{n}\left(Y, V_{i}\right)^{\left(X, K_{i}\right)}$ where $n$ is a positive integer, the $K_{i}$ are compact, and the $V_{i}$ are open sets.

First we show that every member of $\mathcal{B}$ has the homotopy type of a $\mathrm{CW}$ complex. Pick $W=\bigcap_{i=1}^{n}\left(Y, V_{i}\right)^{\left(X, K_{i}\right)} \in \mathcal{B}$. There exists a finite subcomplex $L$ of $X$ such that $\bigcup_{i=1}^{n} K_{i} \subset L$. Since the inclusion $L \subset X$ is a cofibration, the restriction map $R: Y^{X} \rightarrow Y^{L}$ is a fibration. Let $W^{\prime}=\bigcap_{i=1}^{n}\left(Y, V_{i}\right)^{\left(L, K_{i}\right)}$. Then $W=R^{-1}\left(W^{\prime}\right)$ and we obtain a fibration $r=\left.R\right|_{W}: W \rightarrow W^{\prime}$.

Since $L$ is a finite CW complex, it is a metrizable compactum. By Mardešić and Segal [9, Theorem 4 on page 38], the space $Y^{L}$ is an ANR. By Theorem 2.1. therefore, $W^{\prime}$ has the homotopy type of a CW complex.

As just noted, $Y^{L}$ has CW homotopy type. By assumption, so has $Y^{X}$. Therefore by Stasheff [18, Corollary 13], also the fibers of $R$ have CW homotopy type. Note 
that each fiber of $r$ is also a fiber of $R$. Thus both the base space $W^{\prime}$ and all fibers of $r$ have CW homotopy type. By Stasheff [18, Proposition 0], also the total space $W$ has CW homotopy type.

Finally we show that every open set of $Y^{X}$ has the homotopy type of a CW complex. Let $U \subset Y^{X}$ be an arbitrary open set. Then $U$ is covered by the collection of open sets $\mathcal{B}^{\prime}=\{V \in \mathcal{B} ; V \subset U\}$. By definition of $\mathcal{B}$, the collection $\mathcal{B}^{\prime}$ is closed under finite intersections. Since $U$ is metrizable, the open cover $\mathcal{B}^{\prime}$ of $U$ is numerable and each of its members is of CW homotopy type. Then, by tom Dieck 3 , Theorem 4] (note that the assumption there is on finite intersections), $U$ has the homotopy type of a CW complex.

Proof of Lemma 1.5. Assume that $X$ is uncountable and let $\left\{e_{\lambda} ; \lambda \in \Lambda\right\}$ be the collection of all (open) cells of $X$. By assumption, $Y$ admits a path $\gamma: I \rightarrow Y$ such that $\gamma(0) \neq \gamma(1)$. Let $c \in Y^{X}$ denote the constant map with the value $\gamma(0)$. We claim that $Y^{X}$ is not first countable at $c$. Assuming the contrary there exists a countable neighbourhood basis at $c$ which we denote by $\left\{U_{n}\right\}_{n=1}^{\infty}$. As $Y^{X}$ is metrizable, necessarily $\bigcap_{n=1}^{\infty} U_{n}=\{c\}$. As $c$ is constant, there is no loss of generality in assuming that $U_{n}=\left(Y, V_{n}\right)^{\left(X, K_{n}\right)}$, where the $V_{n}$ are open neighborhoods of $\gamma(0)$ in $Y$ and the $K_{n}$ are finite subcomplexes of $X$. Set $K=\bigcup_{n=1}^{\infty} K_{n}$. Then $K$ is a countable subcomplex of $X$ and hence, by assumption, a proper subcomplex of $X$. Fix a point $p \in X \backslash K$. By the Urysohn lemma, there exists $\varphi: X \rightarrow I$ such that $\varphi(p)=1$ and $\varphi(K)=\{0\}$. Let $f=\gamma \circ \varphi$. Obviously $f\left(K_{n}\right)=\{\gamma(0)\} \subset V_{n}$; hence $f \in U_{n}$, for all $n$. But $f \neq c$, a contradiction.

\section{Proof of Theorem 1.2 and Corollary 1.4}

The proof of Theorem 1.2 can be obtained by translating the proof of the Main Theorem in 14 to our setting. We first recall the result of Toruńczyk [19, 20] characterizing $\ell^{2}$-manifolds. To state this result we make some definitions concerning approximations of maps. Given an open cover $\mathcal{U}$ of $M$ we say that maps $f, g: N \rightarrow M$ are $\mathcal{U}$-close if for each $x \in N$ the points $f(x)$ and $g(x)$ belong to a common member of $\mathcal{U}$. If moreover $f$ and $g$ are joined by a homotopy $h: N \times I \rightarrow M$ with $h(\{x\} \times I)$ contained in some member of $\mathcal{U}$ for each $x \in N$, the maps $f$ and $g$ are said to be $\mathcal{U}$-homotopic and $h$ is called a $\mathcal{U}$-homotopy. In the above, assume that $M$ is a metric space and let $\varepsilon>0$. If $\mathcal{U}$ is the collection of all open sets of diameter $<\varepsilon$, we say $\varepsilon$-close, $\varepsilon$-homotopic, and $\varepsilon$-homotopy to mean $\mathcal{U}$-close, $\mathcal{U}$-homotopic, and $\mathcal{U}$-homotopy, respectively.

Let $D$ denote the topological sum $\bigoplus_{n=1}^{\infty} I^{n}$. We say that the space $M$ satisfies the DAP (Discrete Approximation Property) if for any map $f: D \rightarrow M$ and any open cover $\mathcal{U}$ of $M$, there exists a map $g$ which is $\mathcal{U}$-close to $f$ such that the family $\left\{g\left(I^{n}\right)\right\}_{n \in \mathbb{N}}$ of images is discrete in $M$.

Theorem 3.1 (Toruńczyk). A separable completely metrizable space $M$ is an $\ell^{2}$ manifold if and only if it is an ANR and has the DAP.

We use the following criterion, which is easily derived from [4, Remark 2], to check for the DAP.

Lemma 3.2. Let $(M, d)$ be a locally path connected metric space and let $M_{1} \subset$ $M_{2} \subset \cdots \subset M$ be an increasing sequence of subsets of $M$. If $M$ satisfies (i) and (ii), then $M$ satisfies the DAP. 
(i) For any $\varepsilon>0$ and $f: I^{n} \rightarrow M$, there exist $i \geq 1$ and $g: I^{n} \rightarrow M_{i}$ such that $g$ is $\varepsilon$-homotopic to $f$.

(ii) For any $\varepsilon>0$ there exists $\delta>0$ such that for any $i \geq 1$ and $f: I^{n} \rightarrow M_{i}$, there exists $j \geq i$ and $g: I^{n} \rightarrow M_{j}$ which is $\varepsilon$-homotopic to $f$ and satisfies $d\left(f\left(I^{n}\right), g\left(I^{n}\right)\right) \geq \delta$.

Notation 3.3. Let $X$ be a countable CW complex and let $Y, Z$ be spaces. Assume that $Z$ is locally compact. In this situation, a function $f: Z \times X \rightarrow Y$ is continuous if and only if the adjoint $\hat{f}: Z \rightarrow Y^{X}$, defined by $\hat{f}(z)(x)=f(z, x)$, is continuous. In view of this, we shall use the same symbol to denote $f$ and $\hat{f}$.

Proof of Theorem 1.2. If the function space $Y^{X}$ is an $\ell^{2}$-manifold, then it is an ANR; that is, the condition (i) in Theorem 1.1 holds. Conversely, assume that $Y^{X}$ is an ANR. We shall show that $Y^{X}$ is an $\ell^{2}$-manifold. By Theorem 3.1, it is enough to show that $Y^{X}$ satisfies the DAP.

Express $X$ as $X=\bigcup_{m=1}^{\infty} K_{m}$, where each $K_{m}$ is a finite subcomplex of $X$ and $\operatorname{dim} K_{1} \geq 1$. We can take distinct points $x_{1}, x_{2}, \ldots$ and $x_{\infty}$ in $K_{1}$ such that $x_{i} \rightarrow x_{\infty}$ as $i$ tends to infinity. Notice that $Y^{X}$ contains a homeomorphic copy of $Y$ as a retract, which means that $Y$ is an ANR since $Y^{X}$ is by assumption. Using [11, Lemma 2.1] we can choose an admissible metric $d$ of $Y$ with respect to which

(1) for every $\varepsilon>0$ there exists $\delta>0$ such that every two $\delta$-close maps from any space to $Y$ are $\varepsilon$-homotopic,

(2) every path component of $Y$ has diameter $>1$,

(3) $\operatorname{diam} Y<\infty$.

Let $\mathbf{F}$ denote the function space $Y^{X}$. We can define an admissible metric $\bar{d}$ of $\mathbf{F}$ by

$$
\bar{d}(f, g)=\sum_{m=1}^{\infty} 2^{-m} \sup _{x \in K_{m}} d(f(x), g(x)) .
$$

We define a subset $\mathbf{F}_{i}$ of $\mathbf{F}$, where $i \geq 1$, by

$$
\mathbf{F}_{i}=\left\{f \in \mathbf{F} ; f\left(x_{\infty}\right)=f\left(x_{j}\right) \text { for all } j \geq i\right\} .
$$

Then obviously $\mathbf{F}_{i} \subset \mathbf{F}_{i+1}$. We shall show that the sequence $\mathbf{F}_{1} \subset \mathbf{F}_{2} \subset \cdots$ satisfies the conditions (i) and (ii) in Lemma 3.2, which will conclude the proof.

To verify the condition (i), take any $f: I^{n} \rightarrow \mathbf{F}$ and $\varepsilon>0$. By (1) we can take $\delta>0$ such that every two $\delta$-close maps into $Y$ are $\varepsilon$-homotopic. By compactness of $I^{n}$, there exists $i \geq 1$ such that

$$
d\left(f\left(s, x_{k}\right), f\left(s, x_{\infty}\right)\right)<\delta \quad\left(k \geq i, s \in I^{n}\right) .
$$

Since $Y$ is an ANR, by the homotopy extension theorem [13, Theorem 5.1.3] and the choice of $\delta$, there exists an $\varepsilon$-homotopy $h: I^{n} \times X \times I \rightarrow Y$ such that $h(s, x, 0)=$ $f(s, x)\left(s \in I^{n}, x \in X\right)$ and $h\left(s, x_{k}, 1\right)=f\left(s, x_{\infty}\right)\left(s \in I^{n}, i \leq k \leq \infty\right)$. We define $g: I^{n} \rightarrow \mathbf{F}$ by $g(s)(x)=h(s, x, 1)\left(s \in I^{n}, x \in X\right)$. Then we have $g\left(I^{n}\right) \subset \mathbf{F}_{i}$. Moreover, if we set $H(s, t)(x)=h(s, x, t)$, then $H: I^{n} \times I \rightarrow \mathbf{F}$ is a homotopy connecting $f$ with $g$. Then we have

$$
\begin{aligned}
\bar{d}\left(H(s, t), H\left(s, t^{\prime}\right)\right) & =\sum_{m=1}^{\infty} 2^{-m} \sup _{x \in K_{m}} d\left(h(s, x, t), h\left(s, x, t^{\prime}\right)\right) \\
& <\sum_{m=1}^{\infty} 2^{-m} \varepsilon=\varepsilon
\end{aligned}
$$


for $s \in I^{n}$ and $t, t^{\prime} \in I$. This shows that $H$ is an $\varepsilon$-homotopy. The condition (i) is verified.

It remains to verify the condition (ii). Take any $\varepsilon>0$. By (1), there exists $0<\delta \leq 1 / 8$ such that every two $10 \delta$-close maps into $Y$ are $\varepsilon$-homotopic. Take any $i \geq 1$ and $f: I^{n} \rightarrow \mathbf{F}_{i}$. Put $K=f\left(I^{n} \times\left\{x_{j} ; i \leq j \leq \infty\right\}\right) \subset Y$. Since $K$ is compact, there exist $N \geq 1$ and $y_{1}, \ldots, y_{N} \in K$ such that

$$
K \subset \bigcup_{k=1}^{N} B\left(y_{k}, 2 \delta\right),
$$

where $B(y, r)$ denotes an open ball in $Y$. Since each path component of $Y$ has diameter $>1 \geq 8 \delta$ by $(2)$, we can choose a point $z_{k} \in B\left(y_{k}, 6 \delta\right) \backslash B\left(y_{k}, 4 \delta\right)$ for each $1 \leq k \leq N$. Then, by the choice of $\delta$ and the homotopy extension theorem, we have $\varepsilon$-homotopies $h_{k}: Y \times I \rightarrow Y(1 \leq k \leq N)$ such that $h_{k}(y, 0)=y, h_{k}(y, 1)=$ $z_{k}\left(y \in B\left(y_{k}, 4 \delta\right)\right)$ and such that $h_{k}(y, t)=y\left(y \notin B\left(y_{k}, 6 \delta\right)\right)$. Again using the homotopy extension theorem, we have an $\varepsilon$-homotopy $h: X \times Y \times I \rightarrow Y$ such that $h(x, y, 0)=y, h\left(x_{i+k}, y, t\right)=h_{k}(y, t)(1 \leq k \leq N)$ and such that $h\left(x_{j}, y, t\right)=$ $y(i+N<j \leq \infty)$. Finally, we define $g: I^{n} \rightarrow \mathbf{F}$ by

$$
g(s)(x)=h(x, f(s, x), 1) \quad\left(s \in I^{n}, x \in X\right) .
$$

Then, similarly as in the proof of (i), $f, g: I^{n} \rightarrow \mathbf{F}$ are $\varepsilon$-homotopic via a homotopy $H: I^{n} \times I \rightarrow \mathbf{F}$ defined by $H(s, t)(x)=h(x, f(s, x), t)$. It is easy to see that $g \in \mathbf{F}_{j}$ if we let $j=i+N+1$.

Take any $s, s^{\prime} \in I^{n}$. We claim that $\bar{d}\left(f(s), g\left(s^{\prime}\right)\right) \geq \delta$, which means that $\bar{d}\left(f\left(I^{n}\right), g\left(I^{n}\right)\right) \geq \delta$ and hence the condition (ii) is met. First we consider the case where $d\left(f\left(s, x_{\infty}\right), f\left(s^{\prime}, x_{\infty}\right)\right) \geq 2 \delta$. In this case, we have $d\left(f\left(s, x_{\infty}\right), g\left(s^{\prime}, x_{\infty}\right)\right) \geq$ $2 \delta$, since $g\left(s^{\prime}, x_{\infty}\right)=h\left(x_{\infty}, f\left(s^{\prime}, x_{\infty}\right), 1\right)=f\left(s^{\prime}, x_{\infty}\right)$. Since $x_{\infty} \in K_{1}$, we easily see that $\bar{d}\left(f(s), g\left(s^{\prime}\right)\right) \geq \delta$ as claimed. Second we consider the case where $d\left(f\left(s, x_{\infty}\right), f\left(s^{\prime}, x_{\infty}\right)\right)<2 \delta$. Take $k$ such that $f\left(s, x_{\infty}\right) \in B\left(y_{k}, 2 \delta\right)$. Then $f\left(s^{\prime}, x_{\infty}\right)$ $\in B\left(y_{k}, 4 \delta\right)$ and hence $h_{k}\left(f\left(s^{\prime}, x_{\infty}\right), 1\right)=z_{k}$. Then we have

$$
\begin{aligned}
g\left(s^{\prime}, x_{i+k}\right) & =h\left(x_{i+k}, f\left(s^{\prime}, x_{i+k}\right), 1\right) \\
& =z_{k} \notin B\left(y_{k}, 4 \delta\right) .
\end{aligned}
$$

On the other hand, we see that

$$
f\left(s, x_{i+k}\right)=f\left(s, x_{\infty}\right) \in B\left(y_{k}, 2 \delta\right)
$$

since $f(s) \in \mathbf{F}_{i} \subset \mathbf{F}_{i+k}$. Thus we obtain $d\left(f\left(s, x_{i+k}\right), g\left(s^{\prime}, x_{i+k}\right)\right) \geq 2 \delta$. In view of $x_{i+k} \in K_{1}$, we see that $\bar{d}\left(f(s), g\left(s^{\prime}\right)\right) \geq \delta$ as claimed. This completes the proof.

We refer the reader to Maunder [10, Theorem 6.2.25] for a proof of

Lemma 3.4. If $X$ is homotopy equivalent to $X^{\prime}$ and $Y$ is homotopy equivalent to $Y^{\prime}$, then the function spaces $Y^{X}$ and $Y^{\prime X^{\prime}}$ are homotopy equivalent.

We recall the fact that two homotopy equivalent $\ell^{2}$-manifolds are homeomorphic (see Bessaga-Pełczyński [1, page 316]). Now Corollary 1.4 immediately follows from Lemma 3.4 and Theorem 1.2 . 


\section{Applications}

Combining Theorem 1.2 and the results of [16], we have several consequences of interest. A space $X$ is semilocally path connected at $x \in X$ if the path component containing $x$ contains a neighborhood of $x$. If this holds for every $x$ in $X$ (that is, each path component is open), the space $X$ is called semilocally path connected.

Theorem 4.1. For a connected countable $C W$ complex $X$ which is not a point, the following are equivalent.

(i) $\left(S^{1}\right)^{X}$ is an $\ell^{2}$-manifold.

(ii) $\left(S^{1}\right)^{X}$ has the homotopy type of a $C W$ complex.

(iii) $\left(S^{1}\right)^{X}$ is semilocally path connected at some point.

(iv) $H^{1}(X ; \mathbb{Z})$ is finitely generated.

Proof. We have (i) $\Longleftrightarrow$ (ii) by Theorem 1.2. Evidently, (ii) implies (iii). To prove the implication (iii) $\Longrightarrow$ (iv), we assume that (iv) does not hold. Since $X$ is countable, we can express $X$ as $X=\bigcup_{i=1}^{\infty} K_{i}$, where each $K_{i}$ is a finite subcomplex of $X$, and $K_{1} \subset K_{2} \subset \ldots$ For each $i$, by obstruction theory, we have a commutative diagram

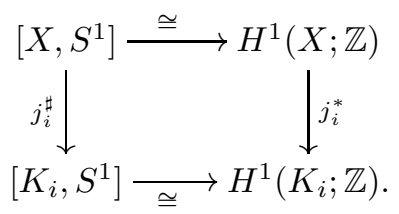

In this diagram, the vertical arrows are induced by the inclusion $j_{i}: K_{i} \subset X$. Notice that $H^{1}\left(K_{i} ; \mathbb{Z}\right)$ is finitely generated, whereas $H^{1}(X ; \mathbb{Z})$ is not by assumption. Since $j_{i}^{*}$ is a homomorphism, the vertical arrow $j_{i}^{*}$ is not injective, which means that every nonempty fiber of $j_{i}^{*}$ contains more than one point. The same is true for $j_{i}^{\sharp}$, for each $i$. This fact implies that $\left(S^{1}\right)^{X}$ is not semilocally path connected at any point, which is the negation of (iii).

Lastly we show that (iv) $\Longrightarrow$ (ii). Assume that (iv) holds. This is to say, $\operatorname{Hom}\left(H_{1}(X), \mathbb{Z}\right) \cong H^{1}(X) \cong \mathbb{Z}^{d}$ for some $0 \leq d<\infty$. By Fuchs [6], $\S 43$, Exercise 10], $H_{1}(X)$ admits a decomposition $H_{1}(X) \cong \mathbb{Z}^{d} \oplus H$ where $\operatorname{Hom}(H, \mathbb{Z})=0$. Now we can appeal to [16, Proposition 4.5.2] to infer (ii).

Alternatively, it follows from the above that the morphisms $H^{1}(X) \rightarrow H^{1}\left(K_{i}\right)$ must be injective for infinitely many $i$. As we can assume the $K_{i}$ to be connected, this implies that $H^{1}\left(X, K_{i}\right)=0$ for infinitely many $i$. Now we can appeal to Theorem 4.2 below.

Theorem 4.2. Let $X$ be a connected countable $C W$ complex which is not a point. Assume that $\operatorname{dim}\left(X \backslash K_{0}\right) \leq n$ for some finite subcomplex $K_{0}$. If for each finite subcomplex $K$ there exists a larger finite subcomplex $L$ such that $H^{i}(X, L ; \mathbb{Z})=0$ for $1 \leq i \leq n$, then the function space $\left(S^{n}\right)^{X}$ is an $\ell^{2}$-manifold.

Proof. If $n \geq 2$, then $\left(S^{n}\right)^{X}$ has the homotopy type of a CW complex by [15, Theorem 2.1]. For $n=1$, the circle $S^{1}$ is actually an Eilenberg-MacLane space $K(\mathbb{Z}, 1)$. The proof of [15, Theorem 3.1] can be generalized easily (in fact abbreviated) to work in this case, too. Theorem 1.2 completes the proof.

Theorem 4.3. Let $X$ be a simply connected, finite-dimensional countable $C W$ complex. Then the following are equivalent. 
(i) For every ANR Y, the space $Y^{X}$ is an ANR.

(ii) For every $C W$ complex $Y$, the space $Y^{X}$ has $C W$ homotopy type.

(iii) For every finite $C W$ complex $Y$, the space $Y^{X}$ is semilocally path connected at some point.

(iv) $X$ has the homotopy type of a finite $C W$ complex.

Proof. The implication (i) $\Longrightarrow$ (ii) follows from Milnor [12, Theorem 2] in conjuction with Theorem 2.1. Next, (ii) $\Longrightarrow$ (iii) is obvious, while (iv) implies (i) by [12, Theorem 3] in conjunction with Lemma 3.4 and Theorem 1.1.

It remains to show (iii) $\Longrightarrow$ (iv). By Hatcher [7, Proposition 4C.1], (iv) is equivalent to $H_{*}(X ; \mathbb{Z})=\bigoplus_{i=1}^{\operatorname{dim} X} H_{i}(X ; \mathbb{Z})$ being finitely generated, since $X$ is simply connected and finite-dimensional. Assume that (iv) does not hold. Then there exists $n$ such that $H_{n}(X, \mathbb{Z})$ is not finitely generated. By the universal coefficient theorem, either $H^{n}(X ; \mathbb{Z})$ or $H^{n+1}(X ; \mathbb{Z})$ is not finitely generated (see Hatcher $[7$, Proposition 3F.12]). Fix $m$ such that $H^{m}(X ; \mathbb{Z})$ is not finitely generated. Since $X$ is finite-dimensional, we can take a connected finite $\mathrm{CW}$ complex such that $\pi_{m}(Y)=\mathbb{Z}$ and $\pi_{i}(Y)=0$ for $0<i \leq \operatorname{dim} X, i \neq m$. Express $X$ as $X=\bigcup_{i=1}^{\infty} K_{i}$, where $K_{i}$ is a finite subcomplex of $X$ for each $i$. By obstruction theory, for each $i$ we have a commutative diagram

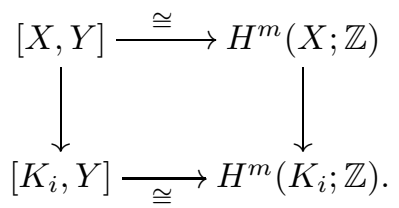

As in the proof of Theorem 4.1, we conclude that the space $Y^{X}$ is not semilocally path connected at any point, which means that (iii) does not hold. This completes the proof.

\section{Acknowledgement}

The first author would like to take this opportunity to thank the second author for bringing the topic of ANR function spaces to his attention.

\section{REFERENCES}

1. C. Bessaga and A. Pełczyński, Selected topics in infinite-dimensional topology, Monografie Matematyczne, Tom 58, Polish Scientific Publishers, Warsaw, 1975. MR0478168 (57:17657)

2. R. Cauty, Une caractérisation des rétractes absolus de voisinage, Fund. Math. 144 (1994), no. 1, 11-22. MR.1271475 (94m:54044)

3. T. tom Dieck, Partitions of unity in homotopy theory, Compos. Math. 23 (1971), 159-167. MR0293625 (45:2702)

4. T. Dobrowolski and H. Toruńczyk, Separable complete ANRs admitting a group structure are Hilbert manifolds, Topology Appl. 12 (1981), 229-235. MR623731 (83a:58007)

5. R. Engelking, General topology (revised and completed ed.), Heldermann Verlag, Berlin, 1989. MR.1039321 (91c:54001)

6. L. Fuchs, Infinite abelian groups, Vol. I, Pure and Applied Mathematics, vol. 36, Academic Press, New York, 1970. MR0255673 (41:333)

7. A. Hatcher, Algebraic topology, Cambridge University Press, 2002. MR.1867354 (2002k:55001)

8. S.-T. Hu, Theory of retracts, Wayne State Univ. Press, Detroit, 1965. MR0181977 (31:6202)

9. Sibe Mardešić, Jack Segal, Shape theory. The inverse system approach, North-Holland Mathematical Library, 26. North-Holland Publishing Co., Amsterdam-New York, 1982. MR 676973 (84b:55020) 
10. C. R. F. Maunder, Algebraic topology, Dover Publications, Inc., Mineola, New York, 1996. MR $1402473(97 \mathrm{c}: 55001)$

11. E. Michael, Uniform ARs and ANRs, Compos. Math. 39 (1979), 129-139. MR546964 (80m:54026)

12. J. Milnor, On spaces having the homotopy type of $C W$-complex, Trans. Amer. Math. Soc. 90 (1959), 272-280. MR0100267(20:6700)

13. J. van Mill, Infinite-dimensional topology: Prerequisites and introduction, North-Holland, Amsterdam, 1989. MR977744 (90a:57025)

14. K. Sakai, The space of cross sections of a bundle, Proc. Amer. Math. Soc. 103 (1988), 956960. MR947690 (90e:57036)

15. J. Smrekar, Compact open topology and CW homotopy type, Topology Appl. 130 (2003), 291-304. MR.1978893 (2004c:55015)

16. J. Smrekar, CW type of inverse limits and function spaces, arXiv:math.AT/07082838.

17. J. Smrekar, Homotopy type of mapping spaces and existence of geometric exponents, Forum Math., in press.

18. J. Stasheff, A classification theorem for fibre spaces, Topology 2 (1963), 239-246. MR0154286 $(27: 4235)$

19. H. Toruńczyk, Characterizing Hilbert space topology, Fund. Math. 111 (1981), 247-262. MR.611763(82i:57016)

20. H. Toruńczyk, A correction of two papers concerning Hilbert manifolds, Fund. Math. 125 (1985), 89-93. MR813992(87m:57017)

21. A. Yamashita, Non-separable Hilbert manifolds of continuous mappings, arXiv:math.GN/ $0610214 \mathrm{v} 1$.

Fakulteta za Matematiko in Fiziko, Jadranska ul. 19, SI-1111 Ljubljana, Slovenia

E-mail address: jaka.smrekar@fmf.uni-lj.si

Graduate School of Mathematical Sciences, The University of Tokyo, 3-8-1, Komaba, Meguro-ku, Tokyo 153-8914, Japan

E-mail address: yonster@ms.u-tokyo.ac.jp 